\title{
Accretion History of Subhalo Population now and then
}

\author{
Carlo Giocoli
}

\author{
Zentrum für Astronomie, ITA, Universität Heidelberg, Albert-Ueberle-Str. 2, 69120 Heidelberg, Germany
}

\begin{abstract}
In the standard model of structure formation galaxies reside in virialized dark matter haloes which extend much beyond the observational radius of the central system. The dark matter halo formation process is hierarchical, small systems collapse at high redshift and then merge together forming larger ones. In this work we study the mass assembly history of host haloes at different observation redshifts and the mass function of accreted satellites (haloes that merge directly on the main halo progenitor). We show that the satellite mass function is universal, both independent on the host halo mass and observation redshift. The satellite mass function also turn out to be universal once only satellites before or after the host halo formation redshift (time at which the main halo progenitor assembles half of its final mass) are considered. We show that the normalizations of these distributions are directly related to the main halo progenitor mass distributions before and after its formation, while their slope and the exponential high mass cut-off remain unchanged.
\end{abstract}

Keywords: Dark Matter, Haloes, Subhaloes, Mass Function, Galaxies

PACS: $98.80-\mathrm{k}$

\section{INTRODUCTION}

Understanding the structure formation process is a fundamental topic in modern cosmology. In the current $\Lambda \mathrm{CDM}$ concordance cosmology, the matter density of the Universe is dominated by cold dark matter (CDM), whose gravitational evolution gives rise to a population of virialized dark matter haloes spanning a wide range of masses [1]. Numerical simulations of structure formation in a CDM universe predict that these dark matter haloes contain a population of subhaloes, which are the remnants of haloes accreted by the host, and which are eroded by the combined effects of gravitational heating and tidal stripping in the potential well of the main halo.

Understanding the evolution of the subhalo mass function, as a function of cosmology, redshift, and host halo mass, is of fundamental importance, with numerous applications. For one, subhaloes are believed to host satellite galaxies, which can thus be used as luminous tracers of the subhalo population. In particular, linking the observed abundances of satellite galaxies to the expected abundance of subhaloes, provides useful insights into the physics of galaxy formation $[2,3,4]$. Studies along these lines indicate that galaxy formation becomes extremely inefficient in low mass haloes, and suggest that there may well be a large population of low mass subhaloes with no optical counterpart $[5,6]$.

In principle, though, these truly 'dark' subhalos may potentially be detected via $\gamma$-ray emission due to dark matter annihilation in their central cores $[7,8,9,10,12,11]$ or via their impact on the flux-ratio statistics of multiply-lensed quasars [13,14]. Alternatively, these techniques may be used to constrain the abundance of subhaloes, which in turn has implications for cosmological parameters and/or the nature of dark matter.

The evolution of the subhalo mass function is also of importance for the survival probability of disk galaxies [15, 16] and even has implications for direct detection experiments of dark matter.

Finally, understanding the rate at which dark matter subhaloes lose mass has important implications for their dynamical friction times, and thus for the merger rates of galaxies [17, 18, 19].

Despite significant progress in the last years, there are still numerous issues that are insufficiently understood. What is the mass function of haloes accreted onto the main progenitor of a present day host halo? Does the distribution depend on the host halo mass and observation redshift? Answareing this question is also very important because, while the mass of the satellite decrease under the gravitational influence of the host due to gravitational heating and tidal stripping, its luminosity is thought to be preserved (author?) [20, 21, 22]. In this work we address these questions using high resolution numerical simulations. We trace back the evolution of host haloes starting from different observation redshifts, $z_{0}=0,0.5,1$ and 2 , identifing progenitor haloes directly accreted onto the main branch. 


\section{SIMULATION AND POST PROCESSING}

Let us consider the outputs of a cosmological N-Body simulation of a $\Lambda$ CDM universe in a periodic cube of side $100 h^{-1} \mathrm{Mpc}$. This simulation has a total number of particles of $400^{3}$, with an individual mass of $m_{p}=1.73 \times$ $10^{9} h^{-1} M_{\odot}$. The cosmological parameters are $\left(\Omega_{m}, \sigma_{8}, h, \Omega_{b} h^{2}\right)=(0.3,0.9,0.70 .0196)$, more details about the GIF2 simulation and the post processing can be found in [23] and [24].

At each simulation snapshot haloes have been identified with the spherical overdensity criterion. Having estimated the density of each particles and sorted them by density, we start from the densest one and grow a sphere of matter around its center, and stop when the mean density within the sphere first fall below the virial value [25]. At this point we assign all particles within the sphere to the newly formed halo, and remove them from the global list. We take the centre of the next halo at the position of the densest particle among the remaining ones and grow a second sphere. We continue in this manner until all particles are assigned to haloes or to the field.

Starting from a given observation redshift $z_{0}$ we build the merging history tree, for all haloes ${ }^{1}$ in the simulation more massive then $10^{11.5} h^{-1} M_{\odot}$, using the halo catalogues at all snapshots, separated by redshift inteval $\mathrm{d} z_{i}$, as follows. Considering a halo with virial mass $M_{z_{0}}$ at $z_{0}$ we define its progenitors at the previous output $z_{1}=z_{0}+\mathrm{d} z_{1}$ to be all haloes contributing with at least $50 \%$ of their particles to the initial system. Among them we call the main halo progenitor the halo providing the largest mass contribution. We repeat the procedure, starting now at $z_{1}$ and considering the progenitor at $z_{2}=z_{1}+\mathrm{d} z_{2}$ of the $z_{1}$-main halo progenitor, and so backward in redshift. In this merging history tree we term satellite all progenitors which, at any time, merge directly on the main progenitor, contributing at least with $50 \%$ of their particles to the initial system at $z=z_{0}$.

In the hierarchical growth of the parent host haloes we identify $z_{f}$, the formation redshift of the halo, to be the redshift at which the main halo progenitor assembles half of its final mass [26]. Because small systems collapse first and merge together we expect more (less) massive haloes to have a lower (higher) formation redshift [27].

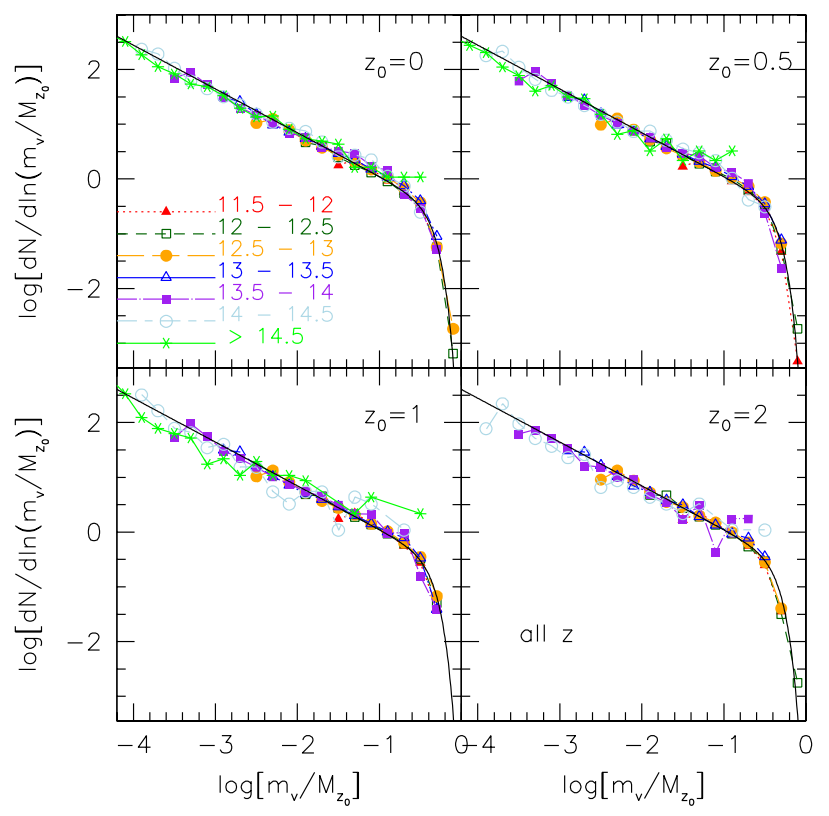

FIGURE 1. Mass accreted in satellites (unevolved subhalo mass function) by the main branch of the tree at all redshifts. In each panel we show the results following the tree starting from different observation redshifts $z_{0}$ and host halo masses. The solid lines represent the fitting function: Equation 1.

\footnotetext{
${ }^{1}$ Haloes which exceed more then $10 \%$ the initial virial mass at any $z>z_{0}$ are not considered in this analysis.
} 


\section{Satellite Mass Function}

In Figure 1 we show the satellite mass function at four different observation redshifts $z_{0}=0,0.5,1$ and 2 , the virial mass of each satellite $m_{\mathrm{v}}$ is expressed in units of the host halo initial mass $M_{z_{0}}$. In each panel different data points and line types refer to various host halo masses. From the figure we notice that all data points, at each observation redshift, are well fitted by the following equation:

$$
\frac{\mathrm{d} N}{\mathrm{~d} \ln \left(m_{v} / M_{z_{0}}\right)}=N_{0} x^{-\alpha} \mathrm{e}^{-6.283 x^{3}}, x=\left|\frac{m_{v}}{\alpha M_{z_{0}}}\right|,
$$

which is represented by the solid curve. The normalization $N_{0}$ and the slope $\alpha$ are respectively equal to 0.21 and 0.8 [23]. This universal distribution has been predicted by [26] using the extendend-Press \& Schechter formalism and linear scale-free power spectrum. [28] found a different slope and normalization following haloes from $z_{0}=0$ to high redshift using a Monte-Carlo merger tree realization of halos in a $\Lambda \mathrm{CDM}$ cosmology However the first measurement of the universality of this distribution in numerical simulation has been done by [23] following haloes only again from $z_{0}=0$. In this work we show that also if we follow haloes starting from different observation redshifts, that are $z_{0} \geq 0$, the distributions still preserve their universality with identical slope and normalization.
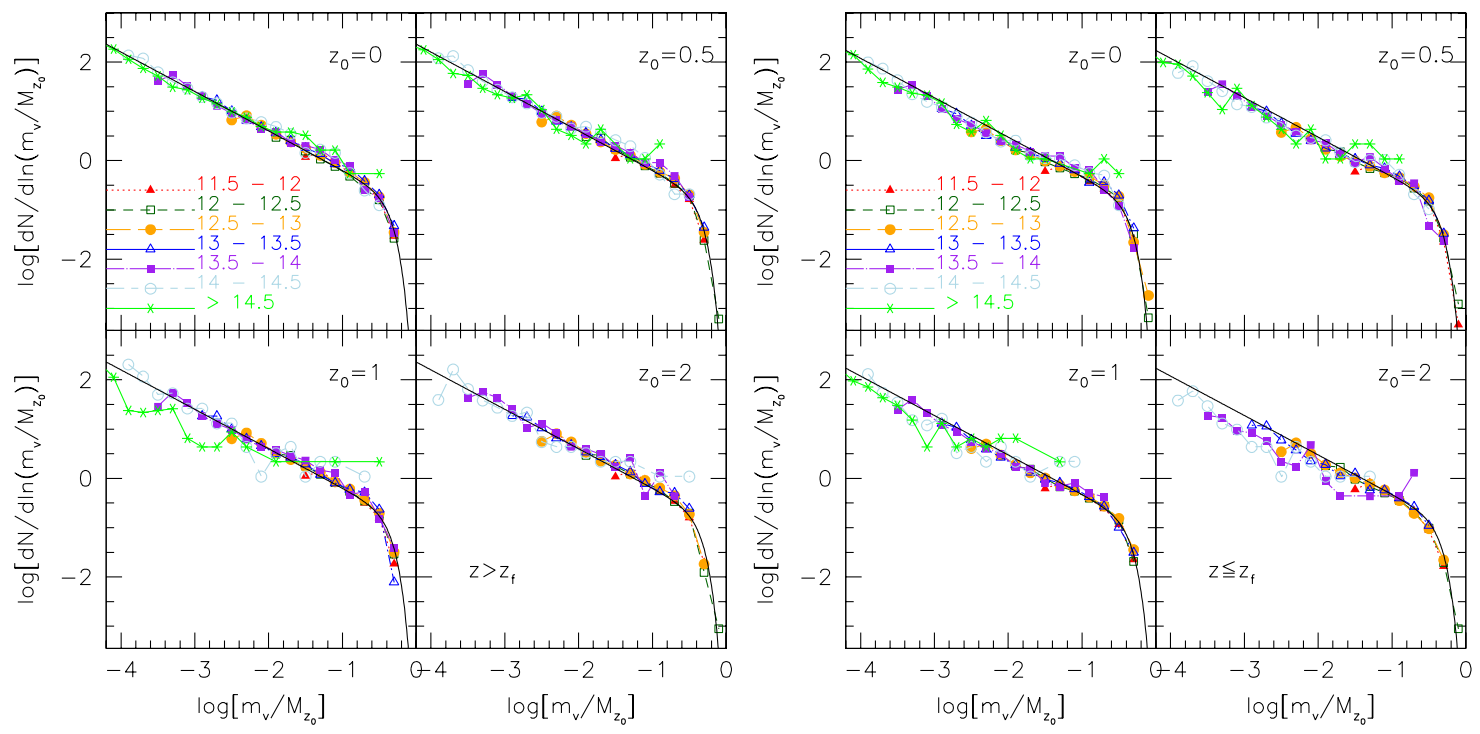

FIGURE 2. Satellite mass function accreted before (left) and after (right) the host halo formation redshift. Different data points and line types refer to varius final host halo masses. The solid curve represent equation 1 with the correct rescaled normalizations.

In Figure 2 we show the satellite mass function for different initial host halo masses and observation redshift, but considering satellite progenitors accreted before (left) and after (right) the formation redshift of the main halo progenitor. Equation 1 also in these cases fits quite well the data point distributions but with different normalizations that we term $N_{0, \mathrm{~b}}\left(\right.$ for $\left.z>z_{f}\right)$ and $N_{0, \mathrm{a}}\left(\right.$ for $\left.z \leq z_{f}\right)$.

\section{Following the Main Branch}

In order to estimate the normalization of the distribution of the satellite mass function before and after the formation redshift of the host halo we need to compute the distribution of the main halo progenitor at $z_{f}$. This is because, if $M_{z_{0}}$ is the host halo mass at $z_{0}$, and all the mass is assembled throught merging of progenitor haloes at $z_{1}$, requiring the mass conservation we can write:

$$
\sum_{i} m_{i, z_{1}}+M_{z_{1}}=M_{z_{0}}
$$


where we divided the sum into the total mass in satellite haloes plus the main halo progenitor. At this point we can also do the same considering the progenitor haloes of $M_{z_{1}}$ at $z_{2}=z_{2}+\mathrm{d} z_{2}$, which gives:

$$
\sum_{i} m_{i, z_{1}}+\sum_{i} m_{i, z_{2}}+M_{z_{2}}=M_{z_{0}}
$$

Following the main halo progenitor back in time until it assembles more then half of its initial mass we can write:

$$
\sum_{j=0}^{z_{j} \leq z_{f}} \sum_{i} m_{i, z_{j}}+\mu M_{z_{0}}=M_{z_{0}}
$$

where $\mu=M_{z_{f}} / M_{z_{0}}$ represents the initial main progenitor mass fraction at $z_{f}$ and $\sum_{j}^{z_{j} \leq z_{f}} \sum_{i} m_{i, z_{j}}$ the total mass accreted in satellites for $z \leq z_{f}$. This first term on the left hand side of Equation 2 is directly related to $N_{0 . a}$, the normalization of the satellite mass function in the right panels of Figure 2.

The analytic estimate of $\mu$ can be obtained considering the distribution of the main halo progenitor mass soon after the formation redshift,

$$
p(\mu) \mathrm{d} \mu=\frac{2}{\pi} \sqrt{\frac{1-\mu}{2 \mu-1}} \frac{\mathrm{d} \mu}{\mu^{2}},
$$

with $1 / 2 \leq \mu \leq 1$, estimated by [29] using the extended-Press \& Schechter formalism.

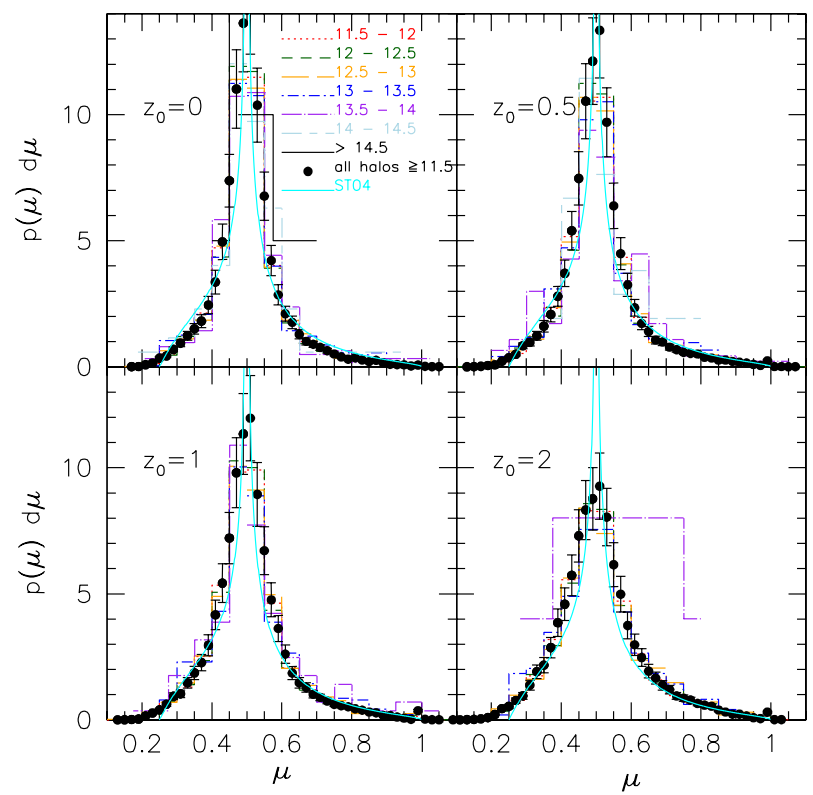

FIGURE 3. Formation mass distribution measured in the GIF2 simulation for different final halo mass bins and observation redshifts with $1 / 2 \leq \mu \leq 1$. The various line type histograms show the result of different final host halo masses. Considering that the distribution does not depend on $M_{0}$, we plot all the halo more massive than $10^{11.5} M_{\odot} / h$ with filled circles. The corresponding error bars assume Poisson counts. For $\mu \leq 1 / 2$ the mass distribution just before the formation is shown. See the main text for more details.

Performing one more step along the main branch of the tree we can re-write Equation 2 and obtain the main halo progenitor distribution soon before its formation:

$$
\sum_{j=0}^{z_{j} \leq z_{f}} \sum_{i} m_{i, z_{j}}+\sum_{i} m_{i, z_{f+1}}+\mu^{\prime} M_{z_{0}}=M_{z_{0}},
$$


where $\mu^{\prime}=M_{z_{f+1}} / M_{z_{0}}$ is the initial main progenitor mass fraction assembled soon before the formation redshift. The $\mu^{\prime}$ distribution has also been estimated by [29] and has the following equation:

$$
p\left(\mu^{\prime}\right) \mathrm{d} \mu^{\prime}=\frac{\mathrm{d} \mu^{\prime} / \mu^{\prime 2}}{\pi\left(1-\mu^{\prime}\right)}\left(\sqrt{\frac{\mu^{\prime}}{1-2 \mu^{\prime}}}-\sqrt{1-2 \mu^{\prime}}\right),
$$

where $1 / 4 \leq \mu^{\prime} \leq 1 / 2$. In this case the mean value of $\mu^{\prime}$ is related to the total mass accreted in satellites for $z>z_{f}$, which is proportional to $N_{0 . b}$, the normalization of the satellite mass function in the left panels of Figure 2 .

In Figure 3 we show the main halo progenitor mass distribution before $(1 / 4 \leq \mu \leq 1 / 2)$ and after $(1 / 2 \leq \mu \leq 1)$ the formation redshift. Histograms with different line types refer to various initial host halo masses at corresponding observation redshifts $z_{0}$ in the GIF2 simulation, while the data points refer to all host haloes considered with mass larger than $10^{11.5} h^{-1} M_{\odot}$. The two solid lines in each panel represent Equations 3 and 4 which perfectly reproduce the simulation results. Mass conservation implies that:

$$
N_{0}=N_{0, \mathrm{a}}+N_{0, \mathrm{~b}}
$$

and for the mean main halo progenitor mass assembled soon before and after the formation redshift:

$$
1=\bar{\mu}^{\prime}+\bar{\mu} .
$$

The normalizations $N_{0, \mathrm{a}}$ and $N_{0, \mathrm{~b}}$ have been estimated measuring the mean halo progenitor mass, for all haloes starting from every observation redshift, soon before and after the formation redshift:

$$
\begin{aligned}
& N_{0, \mathrm{~b}}=\bar{\mu} N_{0}=(0.583 \pm 0.09) N_{0} \\
& N_{0, \mathrm{~b}}=\bar{\mu}^{\prime} N_{0}=(0.432 \pm 0.06) N_{0},
\end{aligned}
$$

absolutely compatible within the error bar with the mass conservation equations above. The small deviation from unity of the sum $\bar{\mu}+\bar{\mu}^{\prime}$ is due to the smooth particle component (mass not in haloes) accreted in numerical simulations along the main branch. In the panels in Figure 2 the solid curves represent the satellite mass function (Equation 1) with the obtained rescaled normalizations that fit quite well the respective data points.

\section{SUMMARY REMARKS}

The standar scenario of structure formation predicts that dark matter haloes grow as consequence of repeated merges events. Central galaxies reside in the main halo progenitor center, while satellite galaxies are in subhaloes. These represent the core of surviving haloes accreted by the main halo progenitor during its cosmic evolution. In these pages we have measured the satellite mass function accreted by the main branch of the merger tree of various host haloes, starting from different observation redshift $z_{0}$ in a numerical N-Body simulation. The satellite mass function turns out to be universal: both independent of the host halo mass and redshift. The shape of the distribution remains unchanged also when satellite haloes accreted before and after the formation redshift are considered. In this case the normalizations can be obtained studying the main halo progenitor distribution soon before and after its formation.

\section{ACKNOWLEDGMENTS}

Thanks to Ravi K. Sheth, Giuseppe Tormen and my fellow travellers in Paris for useful and stimolating discussions, thanks also to Matthias Bartelmann for having careflully read the manuscript.

\section{REFERENCES}

1. Sheth, R. K., \& Tormen, G. 1999, MNRAS, 308, 119

2. Moore, B., Ghigna, S., Governato, F., Lake, G., Quinn, T., Stadel, J., \& Tozzi, P. 1999, ApJL, 524, L19.

3. Bullock, J. S., Kravtsov, A. V., \& Weinberg, D. H. 2000, ApJ, 539, 517

4. Somerville, R. S. 2002, ApJL, 572, L23 
5. Kravtsov, A. V., Berlind, A. A., Wechsler, R. H., Klypin, A. A., Gottlöber, S., Allgood, B., \& Primack, J. R. 2004, ApJ, 609, 35

6. Stoehr, F., White, S. D. M., Tormen, G., \& Springel, V. 2002, MNRAS, 335, L84

7. Stoehr, F., White, S. D. M., Springel, V., Tormen, G., \& Yoshida, N. 2003, MNRAS, 345, 1313

8. Bertone, G. 2006, PhRvD, 73, 103519

9. Pieri, L., Bertone, G., \& Branchini, E. 2008, MNRAS, 384, 1627

10. Giocoli, C., Pieri, L., \& Tormen, G. 2008, MNRAS, 387, 689

11. Giocoli, C., Pieri, L., Tormen, G., \& Moreno, J. 2009, MNRAS, 395, 1620

12. Springel, V., et al. 2008, MNRAS, 391, 1685

13. Metcalf, R. B., \& Madau, P. 2001, ApJ, 563, 9

14. Dalal, N., \& Kochanek, C. S. 2002, ApJ, 572, 25

15. Toth, G., \& Ostriker, J. P. 1992, ApJ, 389, 5

16. Benson, A. J., Lacey, C. G., Frenk, C. S., Baugh, C. M., \& Cole, S. 2004, MNRAS, 351, 1215

17. Benson, A. J., Ellis, R. S., \& Menanteau, F. 2002, MNRAS, 336, 564

18. Zentner, A. R., \& Bullock, J. S. 2003, ApJ, 598, 49

19. Taylor, J. E., \& Babul, A. 2004, MNRAS, 348, 811

20. Vale, A., \& Ostriker, J. P. 2006, MNRAS, 371, 1173

21. Vale, A., \& Ostriker, J. P. 2008, MNRAS, 383, 355

22. Li, R., Mo, H. J., Fan, Z., Cacciato, M., van den Bosch, F. C., Yang, X., \& More, S. 2009, MNRAS, 394, 1016

23. Giocoli, C., Tormen, G., \& van den Bosch, F. C. 2008, MNRAS, 386, 2135

24. Gao, L., White, S. D. M., Jenkins, A., Stoehr, F., \& Springel, V. 2004, MNRAS, 355, 819

25. Eke, V. R., Cole, S., \& Frenk, C. S. 1996, MNRAS, 282, 263

26. Lacey, C., \& Cole, S. 1993, MNRAS, 262, 627

27. Giocoli, C., Moreno, J., Sheth, R. K., \& Tormen, G. 2007, MNRAS, 376, 977

28. van den Bosch, F. C., Tormen, G., \& Giocoli, C. 2005, MNRAS, 359, 1029

29. Sheth, R. K., \& Tormen, G. 2004, MNRAS, 349, 1464 